\title{
Diagnosis and Management of Group a Streptococcal Pharyngitis in the United States, 2011-2015
}

Robert Luo ${ }^{1}$, Joanna Sickler ${ }^{1 *}$, Farnaz Vahidnia ${ }^{2}$, Yuan-Chi Lee ${ }^{2}$, Bianca Frogner ${ }^{3}$ and Matthew Thompson ${ }^{3 *}$

\begin{abstract}
Background: Clinical guidelines for the diagnosis of group A streptococcal (GAS) pharyngitis recommend the use of a rapid antigen detection test (RADT) and/or bacterial culture. This study evaluated the overall diagnosis and treatment of acute pharyngitis in the United States, including predictors of test type and antibiotic prescription.

Methods: A retrospective analysis of pharyngitis events from 2011 through 2015 was conducted using the MarketScan commercial/Medicare databases. A pharyngitis event was defined as occurring within 2 weeks from the index visit. Patient and provider characteristics were examined across 5 testing categories: RADT, RADT plus culture, other tests, nucleic acid amplification testing (NAAT), and no test. Multivariate models were used to identify significant predictors of NAAT use and antibiotic prescription.

Results: A total of 18.8 million acute pharyngitis events were identified in 11.6 million patients. Roughly two-thirds of events (68.2\%) occurred once, and roughly a third of patients (29.1\%) required additional follow-up, but hospitalization was rare (0.3\%). Across all events, $43 \%$ were diagnosed by RADT, while $20 \%$ were diagnosed by RADT plus culture. The proportion of events diagnosed by NAAT increased 3.5-fold from 2011 to 2015 (0.06\% vs 0. 27\%). Antibiotic use was frequent (49.3\%), less often in combination with RADT plus culture (31.2\%) or NAAT alone (34.5\%) but significantly more often with RADT alone (53.4\%) or no test (57.1\%). Pediatricians were significantly less likely than other providers to prescribe antibiotics in their patients, regardless of patient age $(p<0.0001)$.

Conclusions: Antibiotic use for sore throat remains common, with many clinicians not following current guidelines for diagnosis of GAS pharyngitis. Diagnosis of GAS pharyngitis using RADT plus culture or NAAT alone was associated with lower use of antibiotics. Diagnostic testing can help lower the incidence of inappropriate antibiotic use, and inclusion of NAAT in the clinical guidelines for GAS pharyngitis warrants consideration.
\end{abstract}

Keywords: Streptococcal pharyngitis, NAAT, Diagnostics, Antibiotic use

\section{Background}

Acute pharyngitis is a common medical condition that results in an estimated 15 million healthcare visits per year in the United States [1, 2]. Infection with Streptococcus pyogenes (group A beta-hemolytic streptococci) is the most common bacterial cause of acute pharyngitis and is responsible for an estimated 5 to $15 \%$ of sore throat cases among adults [3] and

\footnotetext{
* Correspondence: joanna.sickler@roche.com; mjt@uw.edu

${ }^{1}$ Roche Molecular Systems, Inc, 4300 Hacienda Drive, Pleasanton, CA 94588, USA

${ }^{3}$ Primary Care Innovations Lab, Department of Family Medicine, UW Northgate Clinic, University of Washington, Box 354696, 314 NE Thornton Place, Seattle, WA 98195, USA

Full list of author information is available at the end of the article
}

20 to $30 \%$ of cases among children [2, 4]. Most cases of pharyngitis will resolve on their own without treatment, however, antibiotics are prescribed in approximately $60 \%$ of cases to prevent rare complications (e.g., acute rheumatic fever, rheumatic heart disease, post-streptococcal glomerulonephritis), shorten the duration of illness, prevent the spread of infection to close contacts, and address patient demands [57]. Current treatment guidelines discourage the empirical use of antibiotics for sore throats due to concerns about unnecessary antibiotic exposure and development of resistance.

Accurate diagnosis of group A streptococcal (GAS) pharyngitis by clinical symptoms alone is limited due to the overlap of clinical signs and symptoms between

(C) The Author(s). 2019 Open Access This article is distributed under the terms of the Creative Commons Attribution 4.0 International License (http://creativecommons.org/licenses/by/4.0/), which permits unrestricted use, distribution, and 
bacterial and viral pharyngitis [1]. Current guidelines for diagnosis of GAS pharyngitis in the United States recommend the use of a rapid antigen detection test (RADT) and/or bacterial culture of a throat swab [1]. RADT assays have the benefits of ease of use, rapid turnaround time (< $10 \mathrm{~min}$ ), and high specificity (95\%) but have relatively low sensitivity (70-90\%) [8]. As such, negative RADT results require a confirmatory bacterial culture in pediatric patients, patients at high risk of complications from GAS pharyngitis, and any setting in which clinicians wish to maximize diagnostic sensitivity $[1,8]$. Bacterial culture is both highly sensitive and specific (90-95\%) when performed correctly, but is labor intensive and costly and requires an experienced clinical laboratory to grow and accurately test the bacteria, resulting in reporting delays of 1 to 5 days [9]. Given the low sensitivity rates of RADT and the delays in result reporting (or unavailability) of culture testing, clinicians are often left with the difficult decision of whether or not to prescribe antibiotics when using rapid antigen assays while waiting for confirmatory results, or simply treating (or not treating) the patient without the use of a diagnostic test and accepting any negative clinical consequences or follow-up care.

A number of nucleic acid amplification testing (NAAT) assays for GAS pharyngitis diagnosis have received US Food and Drug Administration (FDA) clearance over the last 3 years. NAAT has shown equivalent sensitivity and specificity to those of bacterial culture [10-15] and improved sensitivity compared with RADT when diagnosing GAS pharyngitis [11]. The potential benefit of using a single assay alone for GAS pharyngitis diagnosis has resulted in some integrated health networks switching to NAAT and implementing rapid transport and reporting mechanisms to reduce turnaround times from days to hours [16]. This facilitates rapid result reporting and timely initiation of appropriate antibiotic therapy, if warranted. Current GAS pharyngitis guidelines do not yet provide guidance on the use of NAAT; however, a recent report from the American Academy of Microbiology suggested that "practice guidelines could inform providers that the nucleic acid tests perform on par with gold standard laboratory testing and encourage their use" [17].

Currently, limited data exist on the factors that impact antibiotic prescribing in GAS pharyngitis. The current study evaluated the diagnosis and treatment of GAS pharyngitis in the United States from 2011 through 2015 in over 11 million patients and investigated the relationship between antibiotic prescribing and provider type, place of service, and GAS pharyngitis diagnostic testing methods. Findings from this study will provide further evidence to inform clinicians and policy makers about which diagnostic tests are routinely used in clinical practice and their impact on antibiotic prescribing for sore throats.

\section{Methods}

Data were compiled from the MarketScan Commercial Claims and Encounters (CCAE) and Medicare Supplemental (MDCR) databases (Truven Health Analytics). The CCAE database contains medical and drug information from employers and health plans, including data on employees and their dependents, whereas the MDCR database includes data on retirees with Medicare supplemental insurance paid by employers [18]. More than 300 employers and 40 private health plans contribute claims information to the MarketScan databases, which is a large-enough data set to provide a nationally representative sample of the American population with employer-sponsored health coverage. The databases have contained data on more than 220 million unique covered individuals from the entire United States since 1995. This analysis used de-identified claims data from inpatient and outpatient visits as well as pharmacies.

From the databases, all patients with a pharyngitis event between January 1, 2011, and December 31, 2015, were identified. Streptococcal pharyngitis was defined by the presence of at least 1 claim during the study period with any International Classification of Diseases, Ninth or Tenth Revision, Clinical Modification diagnostic code for streptococcal sore throat (034.0), acute pharyngitis (462) or acute tonsillitis (463), mapped out to equivalent ICD-10 codes. The index date was defined as the first day of each episode of a pharyngitis claim (index visit) during the study period. A pharyngitis claim (event) was followed for a 2-week period after the index visit (through January $15,2016)$ or disenrollment from the insurance plan, whichever came first. If a patient had a second claim for a pharyngitis visit within 2 weeks after the index visit, it was considered a follow-up to the index event. If a patient had a second claim for a pharyngitis visit more than 2 weeks after the index visit, it was treated as a new event in the analysis. Patients were excluded from the analysis if any condition requiring empirical antibiotic use, including acute bronchitis, cystitis, cellulitis, urethritis, pyelonephritis, diverticulitis, and pneumonia, was documented 2 weeks prior to the index visit (Fig. 1) to remove antibiotic prescriptions not related to the index pharyngitis event.

Descriptive analyses were performed to examine patient and pharyngitis event characteristics, both for the entire cohort and stratified adult (age $\geq 18$ years) and pediatric (age $<$ 18 years) categories. Patient characteristics examined were sex, geographic region of the United States, health plan type, and number of pharyngitis events. Event characteristics included place of service (categorized in the following order: emergency department $>$ urgent care $>$ physician office $>$ laboratory/other, where the first was used if there were multiple places of service associated with an event), provider type (i.e., pediatrician, family physician, internist, and other/ unknown), number of inpatient hospital admissions associated with a pharyngitis event, and medical complications 


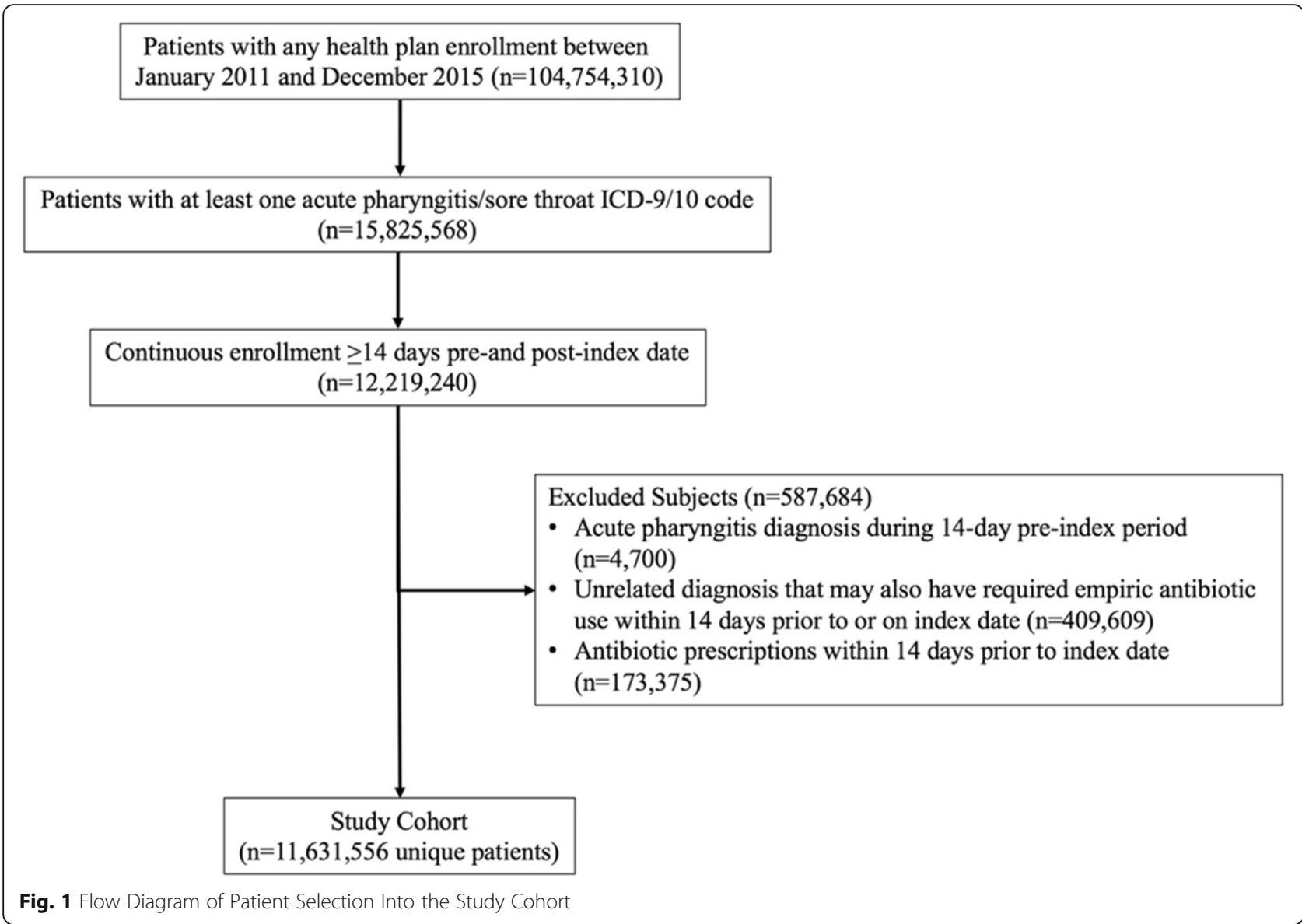

from GAS pharyngitis (i.e., scarlet fever, post-streptococcal glomerulonephritis, rheumatic fever, and streptococcal pneumonia).

GAS pharyngitis diagnostic tests were identified using Current Procedural Terminology (CPT) codes for NAAT (87651), throat culture $(87,081,87,070,87,071)$, RADT (87880), and streptococcal immunoassay (87430). Pharyngitis events were grouped by any GAS pharyngitis diagnostic test claims within 2 weeks after the index visit into the following 5 testing categories: NAAT only, RADT only, RADT plus culture, no test, and other test combination. Other test combination included any combination of the aforementioned test categories with or without streptococcal immunoassay (CPT code: 87430 ). Antibiotic prescription for a pharyngitis event was defined as the presence of a pharmacy claim on or within 2 weeks after the index visit that had a National Drug Code for any of the antibiotics recommended in GAS pharyngitis treatment guidelines [1].

Multivariable models were used to identify pharyngitis event characteristics associated with antibiotic prescription and NAAT use. For each outcome, separate models were constructed for pediatric and adult patients. Adjusted hazard ratios and 95\% confidence intervals were calculated using an Anderson-Gill analysis [19] (SAS version 9.4; SAS
Institute Inc) in a time-dependent model with the following covariates: sex, region, health plan type, calendar year, place of service and provider specialty. Antibiotic prescription models also included diagnostic test type as a covariate. In all models, 0.5 day was added to the follow-up for all events to avoid exclusion of events when a censoring event occurred on the same day as the index visit.

\section{Results}

A total of 104,754,310 patients were identified in the MarketScan databases between January 1, 2011, and December 31, 2015 , of whom 11,631,556 met the criteria for inclusion in the study cohort (Fig. 1). Overall, the study cohort had a mean (SD) age of 24.5 (18.5) years. The majority of patients were female (58.1\%), and $54.1 \%$ of all patients were $\geq 18$ years of age (Table 1). Most patients had only 1 pharyngitis event during the study period (68.2\%). When stratified by age group, a higher proportion of adults than children were female (63.7\% vs. $51.6 \%)$ (Table 1$)$.

Within the entire study cohort, there were 18,778,397 distinct pharyngitis events (Table 2). Adults were more likely than children to have only 1 pharyngitis event $(78.3 \%$ vs. 56.4\%). Roughly one-third of events led to 1 or more follow-up visits (29.1\%), but hospitalization was rare (0.3\%). 
Table 1 Patient characteristics

\begin{tabular}{|c|c|c|c|}
\hline Characteristic & All Ages & Age $<18$ years & Age $\geq 18$ years \\
\hline No. of patients & $11,631,556$ & $5,333,333$ & $6,293,223$ \\
\hline Age, mean (SD), years & $24.5(18.5)$ & $8.4(4.9)$ & $38.0(14.4)$ \\
\hline \multicolumn{4}{|l|}{ Sex, $\%$} \\
\hline Male & 41.9 & 48.5 & 36.3 \\
\hline Female & 58.1 & 51.6 & 63.7 \\
\hline \multicolumn{4}{|l|}{ Region of the United States, \% } \\
\hline Northeast & 17.7 & 18.1 & 17.4 \\
\hline North central & 22.5 & 23.3 & 21.8 \\
\hline South & 42.9 & 42.7 & 43.0 \\
\hline West & 14.7 & 13.5 & 15.8 \\
\hline Unknown & 2.2 & 2.4 & 2.0 \\
\hline \multicolumn{4}{|l|}{ Health plan type, $\%^{\mathrm{a}}$} \\
\hline Managed care (EPO/HMO) & 12.5 & 12.5 & 12.5 \\
\hline Preferred provider (POS/PPO) & 71.0 & 70.5 & 71.5 \\
\hline High deductible (CDHP/HDHP) & 11.6 & 12.0 & 11.3 \\
\hline Unknown & 4.9 & 5.1 & 4.7 \\
\hline \multicolumn{4}{|c|}{ Distinct acute pharyngitis events, 2011-2015, \% } \\
\hline 1 & 68.2 & 56.4 & 78.3 \\
\hline 2 & 18.2 & 21.7 & 15.3 \\
\hline 3 & 6.8 & 10.0 & 4.2 \\
\hline$\geq 4$ & 6.7 & 11.9 & 2.3 \\
\hline
\end{tabular}

${ }^{a}$ Managed care is with an established physician network to receive care: $\mathrm{HMO}$, health maintenance organization, EPO, exclusive provider organization; Preferred provider is with an established physician network and the option to see provider out of the network generally with increased fees: POS, point of service; POS with cap, point of service with capitation; PPO, preferred provider organization; High deductible requires members to pay for services out of pocket until a limit is reached: HDHP, high-deductible health plan. Some include reimbursement from an account before the insurance starts: CDHP, consumer-driven health plan

Overall, antibiotics were used for $49.3 \%$ of events and were prescribed at higher rates among adults (54.4\%) than among children (45.0\%). When information was available on provider specialty type, the most common provider specialties associated with the index visit were pediatrician (28.3\%), family physician (26.2\%), and internist (7.1\%) across all events (Table 2).

The most common place of service for pharyngitis events was the physician office (83.7\%), with urgent care $(7.0 \%)$ and emergency department (3.6\%) being less common. Among all pharyngitis events, diagnosis by RADT was most common (43.0\%), followed by no test (27.9\%) and RADT plus culture (19.8\%). Over the study period, the proportions of events diagnosed using RADT, RADT plus culture, no test, or other tests remained stable (Fig. $2 \mathrm{a}, p<0.001$ ). In contrast, the proportion of events diagnosed using NAAT, while small in overall percentage and number of cases, increased approximately 3.5 -fold, from $0.06 \%$ in $2011(n=2767)$ to $0.27 \%$ in 2015 ( $n=8180)$ (Fig. 2b, b, $p<0.0001)$.

RADT alone was the most common test type used by pediatricians (46.8\%) and family physicians (45.8\%). Pediatricians also had the highest proportion of events (among all events) diagnosed using RADT plus culture
(32.6\%; Additional file 1: Figure S1A, $p<0.001)$. In contrast, internists had the highest proportion of events diagnosed where no test was performed (45.6\%). Similar testing patterns were observed when testing was stratified by place of service (Additional file 1: Figure S1B, $p<0.001$ ), with office-based events showing the highest proportion of RADT only (45.7\%), followed by no test (26.0\%) and RADT plus culture (20.3\%). Events involving the emergency department had no test in the majority of cases (53.7\%). The proportion using NAAT was low $(<0.3 \%)$ among all provider specialties and places of service.

In a multivariable analysis, 28 and $40 \%$ of adult and pediatric NAAT claims, respectively, were associated with a laboratory/other setting $(p<0.0001)$. NAAT was significantly less likely to be used in the emergency department and urgent care ( $p<0.0001$ for both) than in an office setting (Additional file 2: Table S1).

To examine the differences in antibiotic prescription by age group, separate multivariable models were created for adult and pediatric patients. In both models, events diagnosed using RADT alone or no test were significantly associated with increased antibiotic prescription compared with events diagnosed using NAAT or 
Table 2 Pharyngitis event characteristics

\begin{tabular}{|c|c|c|c|}
\hline Characteristic & All Ages & Age $<18$ years & Age $\geq 18$ years \\
\hline No. of events & $18,778,397$ & $10,229,548$ & $8,548,849$ \\
\hline \multicolumn{4}{|l|}{ Provider specialty, $\%$} \\
\hline Pediatrician ${ }^{a}$ & 28.3 & 49.9 & 2.5 \\
\hline Family physician & 26.2 & 15.4 & 39.0 \\
\hline Internist & 7.1 & 2.3 & 12.8 \\
\hline Other/unknown & 38.4 & 32.4 & 45.7 \\
\hline Antibiotics prescribed, $\%$ & 49.3 & 45.0 & 54.4 \\
\hline \multicolumn{4}{|l|}{ Follow-up visits, $\%^{\mathrm{b}}$} \\
\hline 0 & 70.9 & 73.7 & 67.6 \\
\hline 1 & 20.4 & 19.5 & 21.3 \\
\hline$\geq 2$ & 8.7 & 6.8 & 11.0 \\
\hline \multicolumn{4}{|l|}{ Hospital admissions, \% } \\
\hline 0 & 99.7 & 99.8 & 99.6 \\
\hline 1 & 0.3 & 0.2 & 0.4 \\
\hline$\geq 2$ & 0.01 & 0.01 & 0.01 \\
\hline Other complications, $\%^{c}$ & 0.18 & 0.31 & 0.03 \\
\hline
\end{tabular}

${ }^{a}$ Primary care (general practice) physician who specializes in children $(<18$ years)

${ }^{\mathrm{b}}$ Fourteen days post-index visit

'Complications related to streptococcal pharyngitis: scarlet fever, poststreptococcal glomerulonephritis, rheumatic fever, and streptococcal pneumonia

RADT plus culture (Table 3). The majority of the cases diagnosed using RADT plus culture with antibiotic prescriptions had the treatment initiated on the day of the visit (66\%). The use of RADT alone resulted in more antibiotic prescriptions compared with NAAT alone by $49 \%$ among patients aged $\geq 18$ years $(p<0.0001)$ and $123 \%$ among patients aged $<18$ years $(p<0.0001)$.

\section{Discussion}

The current study evaluated the diagnosis and management of acute pharyngitis in the United States from 2011 through 2015 using a database of private and public health insurance claims. With over 11 million adult and pediatric patients with pharyngitis evaluated over the previous 5 years, these data represent one of the largest evaluations of GAS pharyngitis incidence, diagnosis, and antibiotic use to date and offer insights into real-world treatment practices among different types of providers across the United States. As would be expected, the majority of patients were cared for in office settings; family physicians or internists managed the majority of patients aged $\geq 18$ years, and pediatricians managed the majority of patients aged $<18$ years.

The diagnosis of pharyngitis was performed using a wide range of tests among different practitioner types and treatment settings. Overall, RADT alone was used in most cases (43\%), likely given its ease of use and convenience. Additionally, no testing was done more frequently than RADT with confirmatory culture $(27.9 \%$ vs $19.8 \%$, respectively). These findings are in contrast to current clinical guidelines and may reflect real-world clinical practice. For example, clinics and emergency departments may face time pressures, patient demands, and delayed culture results, which may lead them to use RADT only. Alternatively, many cases of pharyngitis may appear obvious to the provider, resulting in no diagnostic testing at all. These real-world situations may contribute to incorrect GAS pharyngitis diagnosis, and consequently inappropriate antibiotic use, given the overlap of symptoms between viral and bacterial causes of pharyngitis. Of interest, while the overall use of NAAT alone was infrequent, the proportion of events diagnosed using NAAT vs. other test types increased 3.5-fold over the study period and roughly doubled in 2014 and again in 2015, coinciding with the approval of GAS pharyngitis NAAT assays.

Similar to results of other studies, almost $50 \%$ of patients in the present study received antibiotics for sore throat/pharyngitis $[6,7,20]$. Given that the literature indicates a $5-30 \%$ prevalence of GAS for pharyngitis $[2,4]$, this confirms high rates of unnecessary antibiotic use. Of interest, cases diagnosed by RADT alone or no test showed significantly greater antibiotic use than those diagnosed by NAAT alone. In contrast, NAAT alone had roughly equivalent rates of antibiotic prescription to those with the current gold standard of RADT plus culture. These results suggest that optimal testing practices that maximize diagnostic sensitivity may lead to more judicious antibiotic prescribing. They also may indicate that providers following current guidelines for diagnosis are more likely to prescribe in line with guidelines recommendations, limiting the empiric use of antibiotics.

FDA-cleared Strep A NAAT assays are now available, including Clinical Laboratory Improvement Amendments-waived PCR systems for use at the point of care (POC) that produce results within 15-25 min, with equivalent sensitivity and specificity to those of reference culture and/or laboratory-based PCR [15, 21]. As such, the use of these assays is possible at POC locations, such as physician offices, emergency departments, and urgent care clinics. Recent research has demonstrated that many patients prefer to avoid unnecessary antibiotics and are willing to undergo diagnostic tests at the POC to guide proper treatment [22]. Our results suggest that these POC NAAT assays could help minimize unnecessary antibiotic prescribing for acute pharyngitis/sore throat by removing the need for confirmatory culture testing, thus reducing the time for (and costs of) result reporting and additional healthcare contacts/calls between the patient and provider. Furthermore, based on the guidelines patients receiving RADT plus culture are likely RADT negative yet $66 \%$ of the antibiotic prescriptions for this group were still initiated at the time of the visit before culture results were available. This further emphasizes the potential value of 


\section{A}

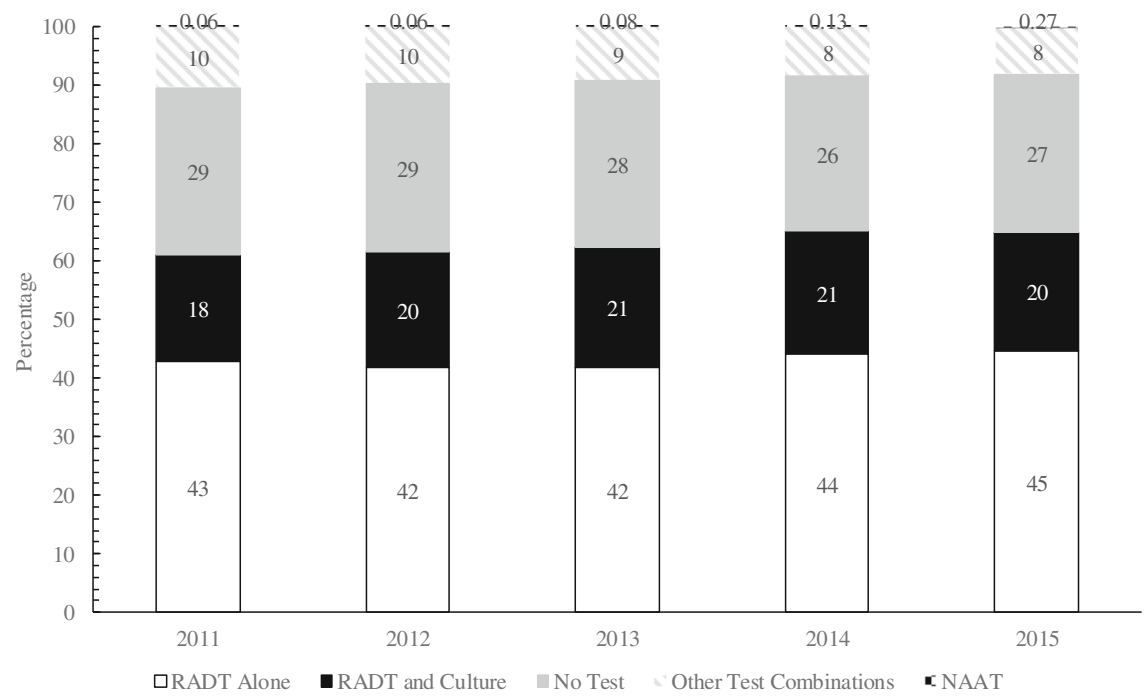

B

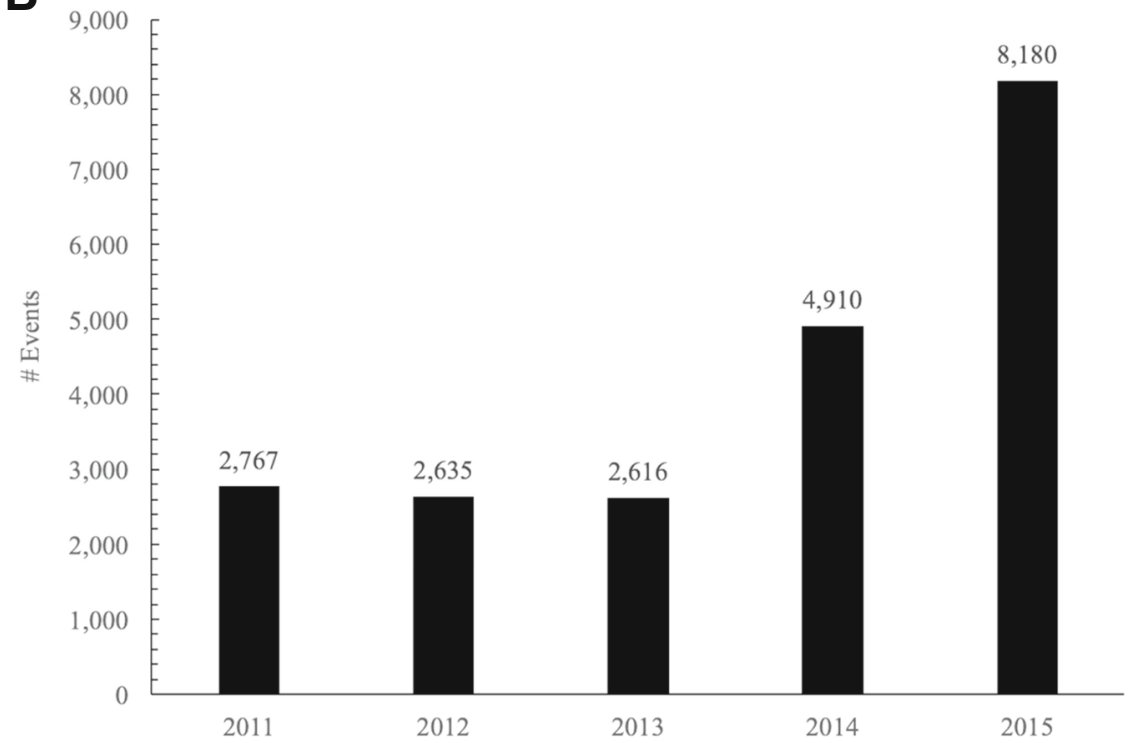

Fig. 2 GAS Testing Patterns by Year: (a) proportions of all test types and (b) number of NAAT tests. NAAT, nucleic acid amplification testing; RADT, rapid antigen detection test

highly sensitive and specific POC NAAT assays to aid clinician diagnosis and reduce empiric antibiotic prescriptions. Diagnostic systems utilizing NAAT technology have demonstrated sensitivity and specificity in clinical trials, but it is important to note that each diagnostic system is unique. Validation of all NAAT is important to ensure performance.

The careful selection of patients based on appropriate clinical symptoms for GAS testing is important regardless of testing modality [23]. A limitation of the analysis is lack of data on the Centor score, so it is unknown what proportion were eligible for GAS testing based on clinical symptoms. Diagnostic tests are always an aid to support clinician decision making. Due to increased sensitivity compared to RADT and even culture, a concern about the use of NAAT is increased detection of colonized patients who do not require treatment, which may theoretically encourage inappropriate antibiotic use of colonized patients. This reinforces the importance of physician assessment to select patients appropriate for GAS testing based on clinical criteria.

Although a strength of the current analysis is the large patient sample and database size, the results are limited by the types of data available and resultant limitations of 
Table 3 Factors associated with antibiotic use among sore throat/pharyngitis visits ${ }^{c}$

\begin{tabular}{|c|c|c|c|c|c|}
\hline & Events (n) & $A B X \%$ & Adjusted $\mathrm{HR}^{\mathrm{b}}$ & \multicolumn{2}{|c|}{ 95\% Confidence Limits } \\
\hline \multicolumn{6}{|l|}{ A. 17 years and younger } \\
\hline \multicolumn{6}{|l|}{ Diagnostic test (ref. NAAT) } \\
\hline RADT only & $4,682,423$ & 52.49 & 2.23 & 2.16 & 2.31 \\
\hline RADT and culture & $2,751,575$ & 26.56 & 0.91 & 0.88 & 0.94 \\
\hline No test & $1,834,351$ & 55.73 & 2.30 & 2.22 & 2.38 \\
\hline Other test combinations & 950,367 & 40.68 & 1.57 & 1.52 & 1.62 \\
\hline \multicolumn{6}{|l|}{ Place of service (ref. office) } \\
\hline ED & 262,364 & 50.15 & 1.01 & 1.00 & 1.01 \\
\hline Urgent care ${ }^{a}$ & 483,304 & 55.71 & 1.15 & 1.15 & 1.16 \\
\hline Laboratory/other & 554,423 & 34.45 & 0.76 & 0.76 & 0.77 \\
\hline \multicolumn{6}{|l|}{ Provider type (ref. pediatrician) } \\
\hline Family medicine & $1,577,559$ & 56.53 & 1.40 & 1.40 & 1.41 \\
\hline Internal medicine & 235,431 & 55.6 & 1.40 & 1.39 & 1.41 \\
\hline Other & $3,309,058$ & 46.52 & 1.20 & 1.20 & 1.21 \\
\hline \multicolumn{6}{|l|}{ B. 18 years and older } \\
\hline \multicolumn{6}{|l|}{ Diagnostic test (ref. NAAT) } \\
\hline RADT only & $3,400,327$ & 54.66 & 1.49 & 1.44 & 1.54 \\
\hline RADT and culture & 967,958 & 44.58 & 1.16 & 1.13 & 1.20 \\
\hline No test & $3,397,174$ & 57.88 & 1.59 & 1.54 & 1.64 \\
\hline Other test combinations & 773,114 & 50.57 & 1.41 & 1.36 & 1.45 \\
\hline \multicolumn{6}{|l|}{ Place of service (ref. office) } \\
\hline ED & 411,369 & 51.01 & 0.92 & 0.91 & 0.92 \\
\hline Urgent care & 824,376 & 57.39 & 1.10 & 1.10 & 1.11 \\
\hline Laboratory/other & 537,543 & 41.82 & 0.73 & 0.72 & 0.73 \\
\hline \multicolumn{6}{|c|}{ Provider type (ref. family medicine) } \\
\hline Internal medicine & 235,431 & 55.6 & 0.99 & 0.99 & 0.99 \\
\hline Pediatrician & $5,107,500$ & 39.92 & 0.65 & 0.65 & 0.66 \\
\hline Other & $3,309,058$ & 46.52 & 0.93 & 0.92 & 0.93 \\
\hline
\end{tabular}

Multivariable Anderson-Gill Survival Models and Adjusted HRs for Antibiotic Use in Patients Aged $(A)<18$ Years and (B) $\geq 18$ Years

${ }^{a}$ Urgent care center's generally see patients on a first-come, first-serve basis for issues that require immediate attention but are not serious enough to warrant an emergency department visit

${ }^{\mathrm{b}}$ Anderson and Gill's Cox Regression Model Counting process using PROC PHREG (Reference)

${ }^{c}$ Adjusted for all variables in the table as well as patient's sex, region, health plan, and calendar year. $P$-values $<0.0001$ for all covariates except for $p=0.0031$ for

ED in patients $<18$

$C l$ confidence interval, ED emergency department, $H R$ hazard ratio, NAAT nucleic acid amplification testing, RADT rapid antigen detection test

the study methods and analyses. We acknowledge that the study population was pulled from a large convenience sample that disproportionately represents the Southern portion of the United States and those with insurance coverage by large employers. As such, our results may reflect regional and/or insurer-type restrictions that may be generalizable only to an employed and insured population. In addition, the data reflect only whether or not the patient received a service (e.g., visit for pharyngitis: yes or no; antibiotic prescribed: yes or no) and do not contain test results or clinical outcomes, which limits the study to one of association and not of causal pathways. As events with only rapid tests could have been from correctly identified positive cases, it is not known what proportion of events with both a rapid test and culture were from negative cases on rapid tests or missed positive cases on rapid tests. Similarly, events with no test may have been clear-cut clinical cases that were either very likely to be GAS pharyngitis or not GAS pharyngitis at all.

\section{Conclusions}

Over a 5-year period in the United States, patient visits for acute pharyngitis events were common, often diagnosed by RADT alone or with no diagnostic test, and resulted in antibiotic prescription in roughly $50 \%$ of cases. Compared with patients $<18$ years of age, patients $\geq 18$ 
years of age were more likely to not receive any diagnostic test and be treated with antibiotics. In addition, NAAT use, while relatively uncommon, increased in frequency over the study period and resulted in antibiotic prescription rates similar to that with RADT plus confirmatory culture testing. Increasing awareness about the impact of antibiotic resistance requires revisiting the role of empirical antibiotic therapy. Diagnostics, in particular sensitive and specific POC diagnostics, can help clinicians to avoid prescribing unnecessary antibiotics. As such, the use of POC NAAT assays or RADT plus confirmatory culture testing may help to limit the inappropriate use of antibiotics and development of bacterial resistance.

These real-world data suggest that clinical guidelines should consider the role of newer diagnostic methods such as NAAT to improve the accuracy of GAS pharyngitis diagnosis as well as stress the overall value of diagnostic use along with clinical symptoms to confirm bacterial infections that require antibiotic treatment.

\section{Additional files}

Additional file 1: Figure S1. GAS Testing by Provider Type: (A) provider specialty and (B) place of service. Other provider types include nurse practitioner/physician assistant, emergency medicine, otolaryngology, multiple providers, and unknown. ED, emergency department; NAAT, nucleic acid amplification testing; RADT, rapid antigen detection test. (DOCX $4476 \mathrm{~kb}$ )

Additional file 2: Table S1. Factors Associated With NAAT Use in Diagnosis of GAS Pharyngitis. Multivariable Anderson-Gill Survival Models and Adjusted HRs for NAAT Use in patients Aged (A) $<18$ Years and $(B) \geq$ 18 Years. (DOCX 17 kb)

\section{Abbreviations \\ CCAE: Commercial claims and encounters; CDHP: Consumer-driven health plan; Cl: Confidence interval; CLIA: Clinical laboratory improvement amendments; CPT: Current procedural terminology codes; Cx: Culture; ED: Emergency department; EPO: Exclusive provider organization; FDA: Food and drug administration; GAS: Group A streptococcus; HDHP: High- deductible health plan; HMO: Health maintenance organization; HR: Hazard ratios; MDCR: Medicare supplemental databases; NAAT: Nucleic Acid amplification testing; PCR: Polymerase chain reaction; POC: Point of care; POS: Point of service; PPO: Preferred provider organization; RADT: Rapid antigen detection test; SD: Standard deviation; US: United States}

\section{Acknowledgments}

The authors would like to thank Andrew D. Luber, PharmD, for expert help during article preparation.

\section{Funding}

This study was funded by Roche Molecular Systems, Inc. The sponsor was responsible for the study design and provided funding for the acquisition of data from Truven Health Analytics (IBM Watson Health), the analysis and interpretation of data and medical writing support for this manuscript.

\section{Availability of data and materials}

The data used for this study are available from MarketScan Commercial Claims and Encounters (CCAE) and Medicare Supplemental (MDCR) databases by Truven Health Analytics (IBM Watson Health) (https:/ truvenhealth.com/markets/life-sciences/products/data-tools/marketscandatabases). The license agreements used to access these databases do not give the authors permission to share these databases.

\section{Authors' contributions}

$\mathrm{RL}, \mathrm{JS}, \mathrm{BF}$ and MT contributed to the preparation and review of the submitted manuscript. FV developed the analysis plan and statistical methods, interpreted the findings, summarized the results and contributed to the writing of the manuscript. YCL assisted the development of statistical analysis plan, conducted data analysis and contributed in writing the manuscript. All authors have read and approved the manuscript for publication.

\section{Ethics approval and consent to participate}

This study utilized deidentified claims data from inpatient and outpatient visits as well as pharmacies. All database records are deidentified in full compliance with US patient confidentiality requirements set forth in the Health Insurance Portability and Accountability Act. Because this study used only deidentified patient records and did not involve the collection, use, or transmittal of individually identifiable data, institutional review board approval to conduct this study was not necessary.

\section{Consent for publication}

Not applicable.

\section{Competing interests}

RL was an employee of Roche during the time of this study and manuscript preparation. JS and FV are employees of Roche. YCL is employed by Genentech, a member of the Roche Group. BF and MT have received consulting fees from Roche. Roche Molecular System's product portfolio includes the cobas Liat Strep A test (a CLIA Waived POC PCR test).

\section{Publisher's Note}

Springer Nature remains neutral with regard to jurisdictional claims in published maps and institutional affiliations.

\section{Author details}

${ }^{1}$ Roche Molecular Systems, Inc, 4300 Hacienda Drive, Pleasanton, CA 94588, USA. ${ }^{2}$ Roche Diagnostics Information Solutions, 4300 Hacienda Drive, Pleasanton, CA 94588, USA. ${ }^{3}$ Primary Care Innovations Lab, Department of Family Medicine, UW Northgate Clinic, University of Washington, Box 354696, 314 NE Thornton Place, Seattle, WA 98195, USA.

Received: 7 June 2018 Accepted: 19 February 2019

Published online: 26 February 2019

\section{References}

1. Shulman ST, Bisno AL, Clegg HW, Gerber MA, Kaplan EL, Lee G, Martin JM, Van Beneden C. Clinical practice guideline for the diagnosis and management of group A streptococcal pharyngitis: 2012 update by the Infectious Diseases Society of America. Clin Infect Dis. 2012;55(10):1279-82.

2. Ebell MH, Smith MA, Barry HC, Ives K, Carey M. The rational clinical examination. Does this patient have strep throat? JAMA. 2000;284(22):2912-8.

3. Centers for Disease Control and Prevention (CDC). Pharyngitis (Strep Throat). 2016. https://www.cdc.gov/groupastrep/diseases-hcp/strep-throat.html. Accessed 12 Sept 2017.

4. Bisno AL. Acute pharyngitis: etiology and diagnosis. Pediatrics. 1996;97(6 pt. 2):949-54.

5. Mangione-Smith R, Elliott MN, Wong L, McDonald L, Roski J. Measuring the quality of care for group A streptococcal pharyngitis in 5 US health plans. Arch Pediatr Adolesc Med. 2005;159(5):491-7.

6. Barnett ML, Linder JA. Antibiotic prescribing to adults with sore throat in the United States, 1997-2010. JAMA Intern Med. 2014:174(1):138-40.

7. Hersh AL, Fleming-Dutra KE, Shapiro DJ, Hyun DY, Hicks LA. Outpatient antibiotic use target-setting workgroup. Frequency of first-line antibiotic selection among US ambulatory care visits for otitis media, sinusitis, and pharyngitis. JAMA Intern Med. 2016;176(12):1870-2.

8. Gerber MA, Shulman ST. Rapid diagnosis of pharyngitis caused by group A streptococci. Clin Microbiol Rev. 2004;17(3):571-580, table of contents.

9. Anjos LM, Marcondes MB, Lima MF, Mondelli AL, Okoshi MP. Streptococcal acute pharyngitis. Rev. Soc Bras Med Trop. 2014;47(4):409-13.

10. Uhl JR, Adamson SC, Vetter EA, Schleck CD, Harmsen WS, Iverson LK, Santrach PJ, Henry NK, Cockerill FR. Comparison of LightCycler PCR, rapid antigen immunoassay, and culture for detection of group A streptococci from throat swabs. J Clin Microbiol. 2003;41(1):242-9. 
11. Wang F, Tian Y, Chen L, Luo R, Sickler J, Liesenfeld O, Chen S. Accurate detection of Streptococcus pyogenes at the point of care using the cobas Liat Strep A nucleic acid test. Clin Pediatr (Phila). 2017;56(12):1128-34.

12. Cohen DM, Russo ME, Jaggi P, Kline J, Gluckman W, Parekh A. Multicenter clinical evaluation of the novel Alere i Strep A isothermal nucleic acid amplification test. J Clin Microbiol. 2015;53(7):2258-61.

13. Uphoff TS, Buchan BW, Ledeboer NA, Granato PA, Daly JA, Marti TN. Multicenter Evaluation of the Solana Group A Streptococcus Assay: Comparison with Culture. J Clin Microbiol. 2016;54(9):2388-90.

14. Premarket notification, $510(\mathrm{k})$ : regulatory requirements for medical devices. Rockville, Md.: Washington, D.C.: U.S. Dept. of Health and Human Services, Public Health Service, Food and Drug Administration, Center for Devices and Radiological Health. K172402 (ARIES Group A Strep Assay). https://www. accessdata.fda.gov/cdrh_docs/reviews/K172402.pdf. Accessed 10 Aug 2018.

15. Premarket notification, $510(\mathrm{k})$ : regulatory requirements for medical devices Rockville, Md.: Washington, D.C.: U.S. Dept. of Health and Human Services, Public Health Service, Food and Drug Administration, Center for Devices and Radiological Health. K172126 (Xpert Xpress Strep A) https:/www. accessdata.fda.gov/cdrh_docs/reviews/K172126.pdf. Accessed 10 Aug 2018.

16. Pritt BS, Patel R, Kirn TJ, Thomson RB. Jr. Point-Counterpoint: A nucleic acid amplification test for Streptococcus pyogenes should replace antigen detection and culture for detection of bacterial pharyngitis. J Clin Microbiol. 2016;54(10):2413-9.

17. Dolen V, Bahk K, Carroll KC, Klugman K, Ledeboer NA, Miller MB. American Academy of Microbiology. Changing diagnostic paradigms for microbiology. 2017.

18. Hansen $L G$, Chang $S$. Health research data for the real world: The MarketScan databases (White Paper); 2016.

19. Anderson PK, Gill RD. Cox's regression model for counting processes: a large sample study. 1982;10(4):1100-20.

20. Nakhoul GN, Hickner J. Management of adults with acute streptococcal pharyngitis: minimal value for backup strep testing and overuse of antibiotics. J Gen Intern Med. 2013;28(6):830-4.

21. Uhl JR, Patel R. Fifteen-Minute Detection of Streptococcus pyogenes in throat swabs by use of a commercially available point-of-care PCR assay. J Clin Microbiol. 2016;54(3):815-5 Epub 2016 Jan 13.

22. Schwartz M, Hardy V, Keppel GA, Alto W, Hornecker J, Robitaille B, Neher J Holmes J, Dirac MA, Cole AM, Thompson M. Patient willingness to have tests to guide antibiotic use for respiratory tract infections: from the WWAMI Region Practice and Research Network (WPRN). J Am Board Fam Med. 2017;30(5):645-56.

23. Tanz RR, Zheng XT, Carter DM, Steele MC, Shulman ST. Caution Needed: Molecular Diagnosis of Pediatric Group A Streptococcal Pharyngitis. J Pediatric Infect Dis Soc. 2018:7(3):e145-7.

Ready to submit your research? Choose BMC and benefit from:

- fast, convenient online submission

- thorough peer review by experienced researchers in your field

- rapid publication on acceptance

- support for research data, including large and complex data types

- gold Open Access which fosters wider collaboration and increased citations

- maximum visibility for your research: over $100 \mathrm{M}$ website views per year

At $\mathrm{BMC}$, research is always in progress.

Learn more biomedcentral.com/submissions 